\title{
Nadolschi Chaotic Systems' Synchronization by Generalized Predictive Control
}

\author{
Mahdi Yaghoobi, PhD \\ Professor of Department of Electrical Engineering \\ Azad University of Mashhad
}

\author{
Alireza Atharian \\ Graduate Student of Azad University of Khomeini \\ Shahr
}

\begin{abstract}
Nadolschi chaotic system is a system with intense non-linear behavior; chaotic systems are stable systems with unique behavioral characteristics. Very high sensitivity to parameters, the initial con ditions and pseudo-random behavior while having a simple structure are examples of these features. Considering numerous applications in various fields, including the issue of encryption and secure communication, and also due to the interesting mathematical aspect of this topic, chaos synchronization has been focused by many researchers in the past two decades. $[9,10]$
\end{abstract}

The aim of this article is Nadolschi chaotic systems' synchronization. To achieve this goal, differences resulting from the synchronization of two similar Nadolschi chaotic systems are obtained. By obtaining this system, synchronization problem is converted into stabilization of fault system resulting from synchronization of two Nadolschi chaotic systems. To achieve this purpose, controller is designed at two levels; first some statements of fault system related to the internal variables are removed by a controller and in the second level, generalized predictive controller will be designed for other variables in the fault system.

The obtained simulation results show that the generalized predictive control has well-managed the operation of tracking with minimum error and so it is very convenient to be used in systems where tracking is important.

\section{Keywords}

Synchronization, Nadolschi Chaotic System, Generalized Predictive Control.

\section{INTRODUCTION}

Chaos is a very interesting phenomenon in terms of nonlinear systems. The discovery of chaos has questioned this basic knowledge principle that certain systems have predictable behavior.

In 1990, Carroll and Pecora proposed the idea of chaos synchronization [1]. Chaotic systems have several remarkable properties such as being ergodic, random, non-periodic, sensitive to initial conditions and unpredictable, all of which make them suitable to be used in cryptography. The researchers argue that these properties can meet several requirements such as dispersion and clutter which are both needed in modern cryptography $[2,3,4,5]$.

Recently, researchers have shown that chaos can useful in many industries such as lasers, biology, economics, chemical reactions, and secure communications [11]. In recent decades, developing cryptographic systems based on chaos theory has attracted a lot of attention.

Although chaos has many benefits but it is necessary to control it. So far many different methods have been proposed to control the chaos that have usually been studied with the aimed of sustainability of the balance points of chaos system.

\section{THE RESEARCH PROBLEM}

Nadolschi system is a system with severe non-linear behavior and in certain cases it has chaotic properties. The purpose of this paper is to synchronize two similar Nadolschi chaos systems but with different initial conditions.

The model of this system is introduced through the following equation:

$$
\left\{\begin{array}{c}
\dot{x}_{1}(t)=-x_{2}(t) x_{3}(t)+a x_{1}(t) \\
\dot{x}_{2}(t)=x_{1}(t) x_{3}(t)+b x_{2}(t) \\
\dot{x}_{3}(t)=x_{1}(t) x_{2}(t) / 3+c x_{3}(t)
\end{array}\right.
$$

Where: $\mathrm{X} 1(\mathrm{t}), \mathrm{x} 2(\mathrm{t})$ and $\mathrm{x} 3(\mathrm{t})$ are state variables and $\mathrm{a}, \mathrm{b}$ and $\mathrm{c}$ are the system parameters; the above system is chaotic when the chosen parameters are particular.

For example, when the selected parameters are $\mathrm{a}=5, \mathrm{~b}=-10$ and $\mathrm{c}=-3.8$, the system is supposed to be chaotic.

The second system which is supposed to be synchronous with the first system is introduced through the following equations:

$$
\left\{\begin{array}{c}
\dot{y}_{1}(t)=-y_{2}(t) y_{3}(t)+a y_{1}(t)+u_{1}(t) \\
\dot{y}_{2}(t)=y_{1}(t) y_{3}(t)+b y_{2}(t)+u_{2}(t) \\
\dot{y}_{3}(t)=y_{1}(t) y_{2}(t) / 3+c y_{3}(t)+u_{3}(t)
\end{array}\right.
$$

Where: $\mathrm{Y} 1(\mathrm{t}), \mathrm{Y} 2(\mathrm{t})$ and $\mathrm{Y} 3(\mathrm{t})$ are state variables and $\mathrm{u} 1(\mathrm{t})$, $\mathrm{u} 2(\mathrm{t})$ and $\mathrm{u} 3(\mathrm{t})$ are the designed data.

As stated in the abstract, the purpose of this research is to synchronize the above mentioned systems. The strategy that has been used for synchronization is the calculation of error signal resulted from synchronization in the first phase and stabilization of error signal in the later stage. In this situation the system for which a controller is designed is fault system and the aim of the controller is to minimize the error rate up to zero. If the error rate comes to zero, synchronization operation has been performed correctly. Therefore all control tasks will be done on the fault system.

Error equation is:

$$
e_{i}(t)=x_{i}(t)-y_{i}(t)
$$

And fault system resulting from synchronization is calculated as follows: 


$$
\left\{\begin{array}{c}
\dot{e}_{1}(t)=a e_{1}(t)-e_{2}(t) e_{3}(t)-y_{2}(t) e_{3}(t) \\
-y_{3}(t) e_{2}(t)-u_{1}(t) \\
\dot{e}_{2}(t)=b e_{2}(t)+e_{1}(t) e_{3}(t)+y_{1}(t) e_{3}(t) \\
\quad+y_{3}(t) e_{1}(t)-u_{2}(t) \\
\dot{e}_{3}(t)=c e_{3}(t)+e_{1}(t) e_{2}(t) / 3+y_{1}(t) e_{2}(t) / 3 \\
+y_{2}(t) e_{1}(t) / 3-u_{3}(t)
\end{array}\right.
$$

At the end of fault system there are $u 1(t), u 2(t)$ and $u 3(t)$ controllers which direct the fault system to zero.

\section{DESIGNING GPC CONTROLLER FOR THE SYNCHRONIZATION OF TWO SIMILAR NADOLSCHI CHAOTIC SYSTEMS}

Controller equations are introduced as below:

$$
\left\{\begin{array}{l}
u_{1}(t)=u_{1_{1}}(t)+u_{1_{2}}(t) \\
u_{2}(t)=u_{2_{1}}(t)+u_{2_{2}}(t) \\
u_{3}(t)=u_{3_{1}}(t)+u_{3_{2}}(t)
\end{array}\right.
$$

As it can be observed controllers have two parts. Th e first part is obtained by an equation and for the second part generalized predictive controller is designed.

Part of the fault system is the parameters related to the fault and the other part is parameters related to the second system. Since the second system is available, the second part can be removed from the fault system as follows, this could be a part of the controller that is called $u_{1_{1}}(t), u_{2_{1}}(t)$ and $u_{3_{1}}(t)$

$$
\left\{\begin{array}{c}
u_{1_{1}}(t)=-y_{2}(t) e_{3}(t)-y_{3}(t) e_{2}(t) \\
u_{2_{1}}(t)=y_{1}(t) e_{3}(t)+y_{3}(t) e_{1}(t) \\
u_{3_{1}}(t)=y_{1}(t) e_{2}(t) / 3+y_{2}(t) e_{1}(t) / 3
\end{array}\right.
$$

Therefore fault system can be rewritten as follows:

$$
\left\{\begin{array}{c}
\dot{e}_{1}(t)=5 e_{1}(t)-e_{2}(t) e_{3}(t)-u_{1_{2}}(t) \\
\dot{e}_{2}(t)=-10 e_{2}(t)+e_{1}(t) e_{3}(t)-u_{2_{2}}(t) \\
\dot{e}_{3}(t)=-3.8 e_{3}(t)+e_{1}(t) e_{2}(t) / 3-u_{3_{2}}(t)
\end{array}\right.
$$

In order to determine system linear behavior around the equilibrium point, first Jacobian matrix is obtained through the following equation:

$$
\mathrm{J}=\left[\begin{array}{ccc}
5 & -e_{3} & -e_{2} \\
e_{3} & -10 & e_{1} \\
\frac{e_{2}}{3} & \frac{e_{1}}{3} & -3.8
\end{array}\right]
$$

The equilibrium for the above system is obtained as follows:

$$
\begin{gathered}
5 e_{1}(t)-e_{2}(t) e_{3}(t)=0, \\
-10 e_{2}(t)+e_{1}(t) e_{3}(t)=0, \\
-3.8 e_{3}(t)+\frac{e_{1}(t) e_{2}(t)}{3}=0,
\end{gathered}
$$

Now, by setting Jacobian matrix at equilibrium $(0,0,0)$, its behavior will be replaced by following linear equation [8].

$$
\begin{gathered}
x=A \dot{x}+B u \\
y=C x
\end{gathered}
$$

$\mathrm{A}=\left[\begin{array}{ccc}5 & 0 & 0 \\ 0 & -10 & 0 \\ 0 & 0 & -3.8\end{array}\right], \mathrm{B}=\left[\begin{array}{ccc}-1 & 0 & 0 \\ 0 & -1 & 0 \\ 0 & 0 & -1\end{array}\right], \mathrm{C}=$

$$
\left[\begin{array}{lll}
1 & 0 & 0 \\
0 & 1 & 0 \\
0 & 0 & 1
\end{array}\right]
$$

The first step in designing a predictive controller is to determine the predictive model. In GPC predictive controller, the predictive model is calculated based on system transfer function. Therefore, to determine the fault system predictive model resulted from synchronization of Nadolschi chaotic systems based on status matrix of the system, the transfer function is calculated according to equation (3-8) as follows.

$G=C(\mathrm{SI}-\mathrm{A})^{-1} B$

$$
\left[\begin{array}{ccc}
\frac{-1}{s-5} & 0 & 0 \\
0 & \frac{-1}{s+10} & 0 \\
0 & 0 & \frac{-5}{5 s+19}
\end{array}\right]
$$

To determine GPC controller parameters such as sampling period, the dominant mode of the system should be determined. As it can be observed the behavior of the function is formed by $\mathrm{P}=0.1$ mode. The settling time parameters (settling Time) and (Rise Time) are:

$\mathrm{ts}=0.4 \mathrm{sec}, \operatorname{tr}=0.22 \mathrm{sec}$

When sampling from transfer function, the closed loop is selected. Since the closed loop transfer function and specifications are not available, the time of sampling is usually selected between $0.1 \tau$ e and $0.05 \tau$ e. Constant time $\tau$ e is equal to controlled system which is defined through following equation: 
$\tau_{e} \approx \sqrt{\frac{1}{(-5)^{2}}+\frac{1}{(10)^{2}}+\frac{1}{\left(\frac{19}{5}\right)^{2}}}=0.3454$

So the right time to take samples is between $0.0172<\mathrm{Ts}$ $<0.03454$ seconds. To obtain control and predictive horizons, the following equations are used:

$$
M=\frac{t_{r}}{T_{s}} \quad P=\frac{t_{s}}{T_{s}}
$$

As a result

$\mathrm{P}=16$,

$\mathrm{M}=8$,

$\mathrm{Ts}=0.025$

\section{SIMULATION RESULTS, CONCLUSIONS AND RECOMMENDATIONS}

In this section, the results of the simulation are provided using Matlab software as discussed below.

\section{Dynamic of Nadolschi chaotic systems}

As described in the first section in the equation (1-2), dynamic equation of Nadolschi chaotic system for the first system is as follows.

$$
\left\{\begin{array}{c}
\dot{x}_{1}(t)=-x_{2}(t) x_{3}(t)+a x_{1}(t) \\
\dot{x}_{2}(t)=x_{1}(t) x_{3}(t)+b x_{2}(t) \\
\dot{x}_{3}(t)=x_{1}(t) x_{2}(t) / 3+c x_{3}(t)
\end{array}\right.
$$

$x(0)=\left[\begin{array}{c}-10 \\ 5 \\ -3\end{array}\right]$

Nadolschi chaotic system dynamic equation for the second system in (2-2) equation is as follows.

$$
\left\{\begin{array}{c}
\dot{y}_{1}(t)=-y_{2}(t) y_{3}(t)+a y_{1}(t) \\
\dot{y}_{2}(t)=y_{1}(t) y_{3}(t)+b y_{2}(t) \\
\dot{y}_{3}(t)=y_{1}(t) y_{2}(t) / 3+c y_{3}(t)
\end{array}\right.
$$

The initial conditions of the first system are:

$$
y(0)=\left[\begin{array}{l}
-5 \\
10 \\
-8
\end{array}\right]
$$

Fault chaotic system dynamic equation resulted from synchronization of the first and second Nadolschi chaotic systems are eventually rewritten as follows:

$$
\left\{\begin{array}{c}
\dot{e}_{1}(t)=5 e_{1}(t)-e_{2}(t) e_{3}(t) \\
\dot{e}_{2}(t)=-10 e_{2}(t)+e_{1}(t) e_{3}(t) \\
\dot{e}_{3}(t)=-3.8 e_{3}(t)+e_{1}(t) e_{2}(t) / 3
\end{array}\right.
$$

$e(0)=\left[\begin{array}{c}-5 \\ -5 \\ 5\end{array}\right]$

The initial conditions of the first system are:

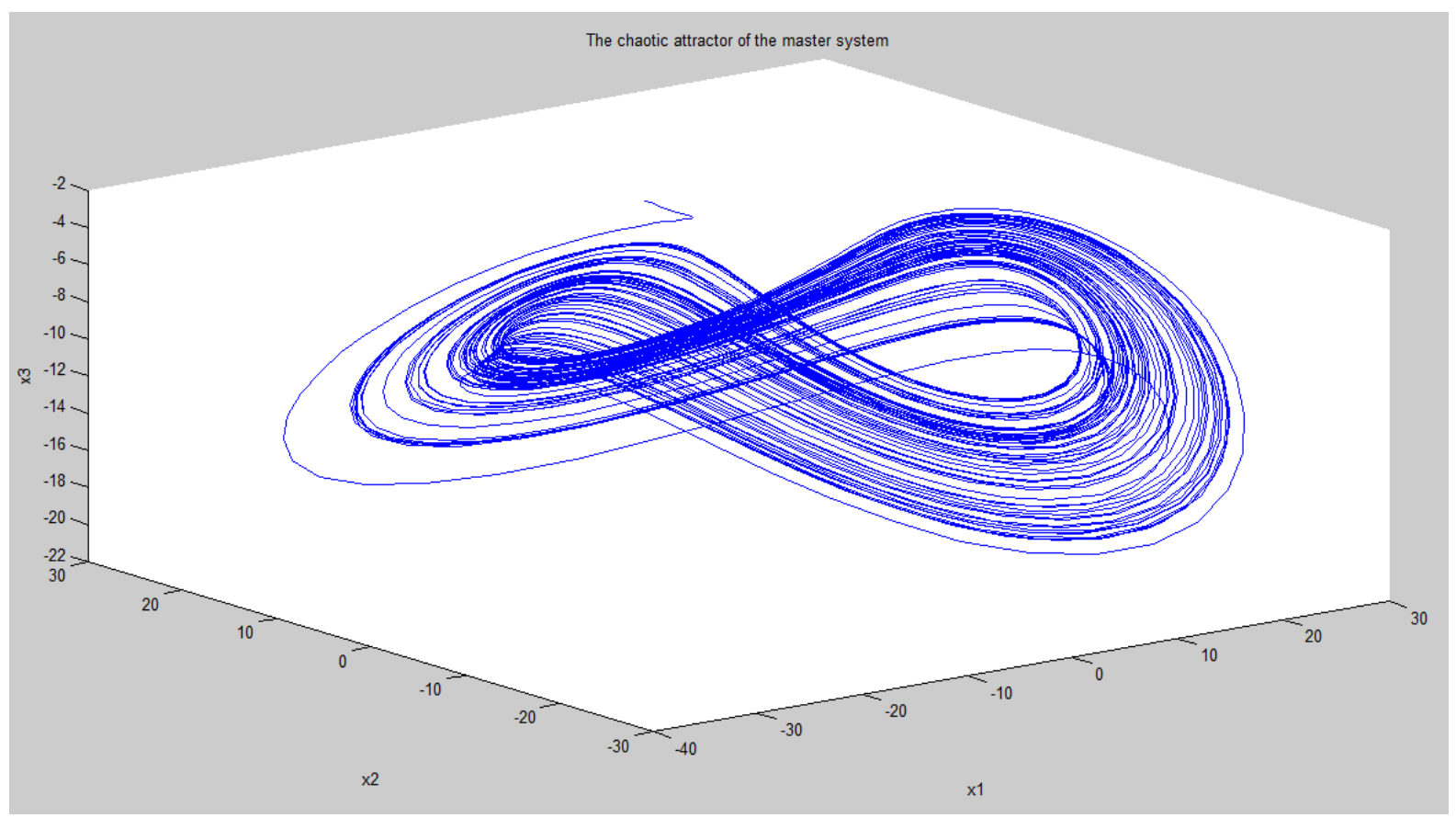

Figure 1: The chaotic attractor of the drive syste 


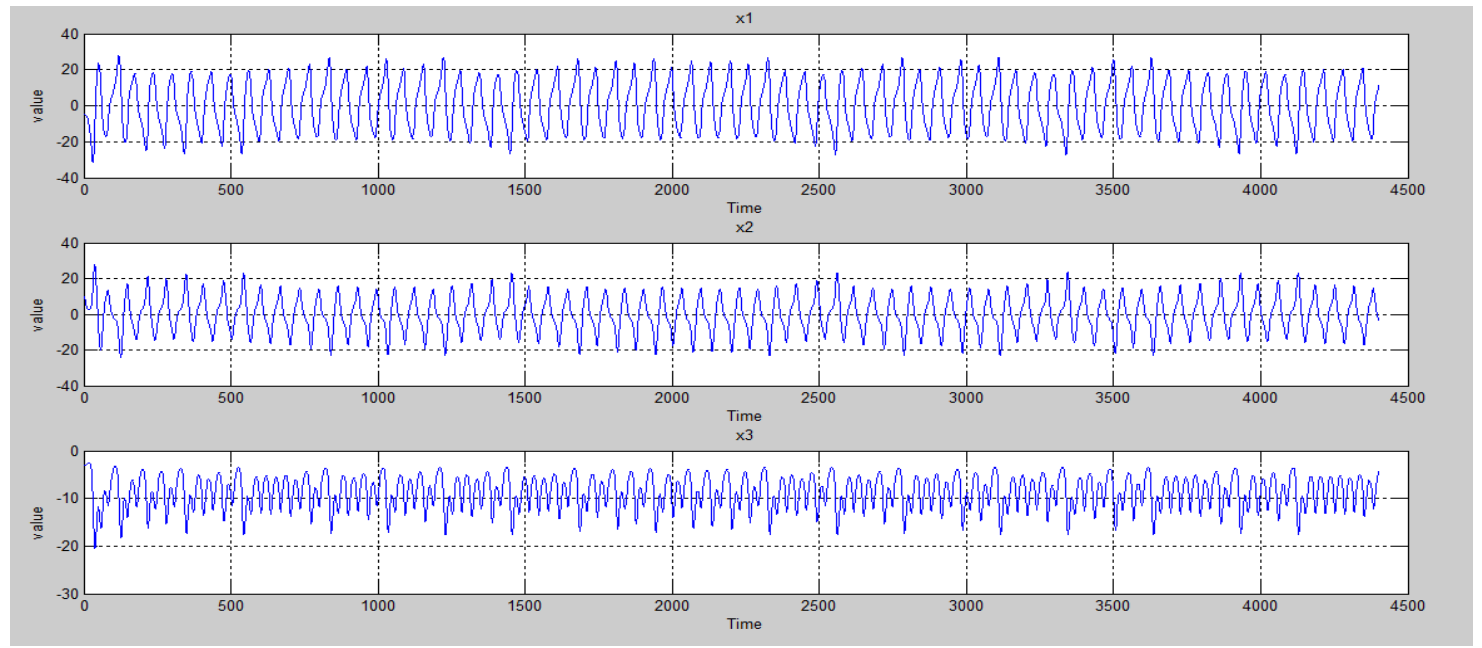

Figure 2: The Answer state chaotic attractor of the drive system

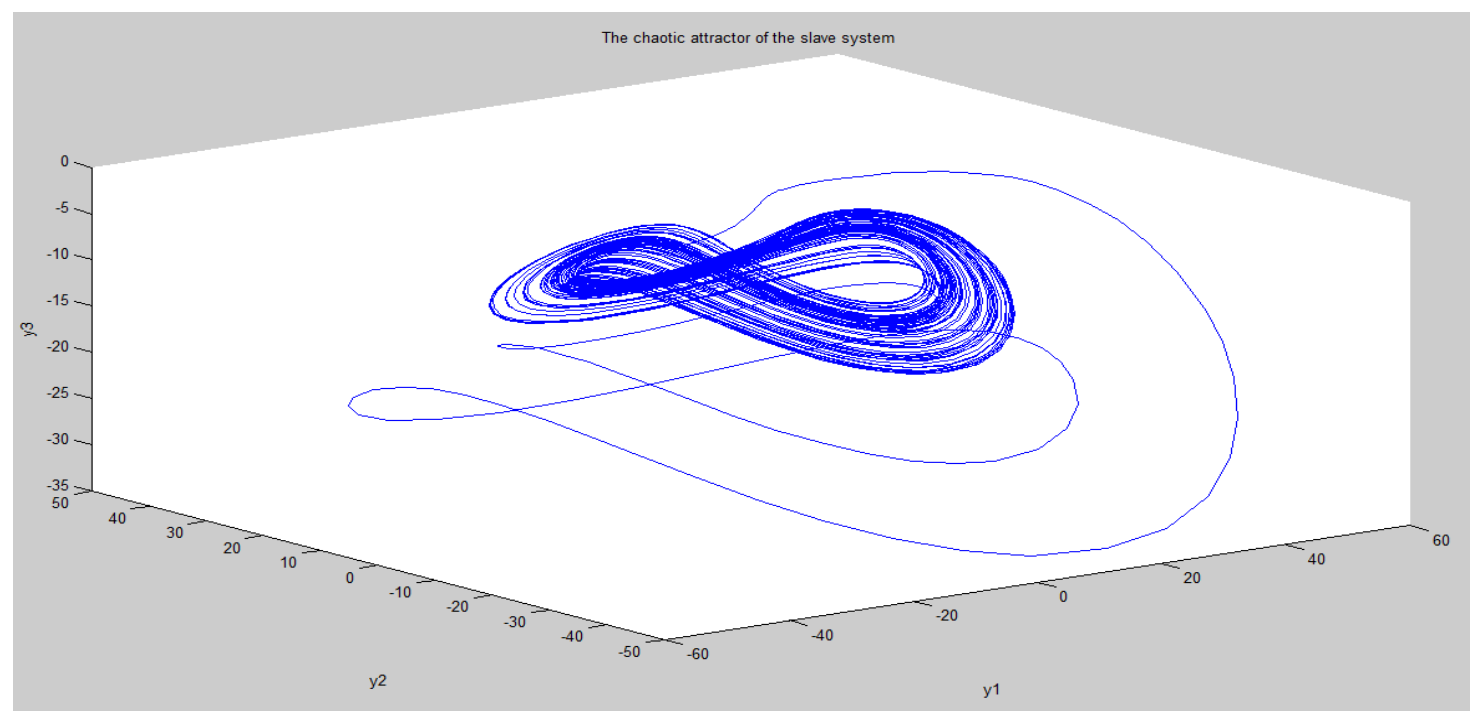

Figure 3: The chaotic attractor of the response system

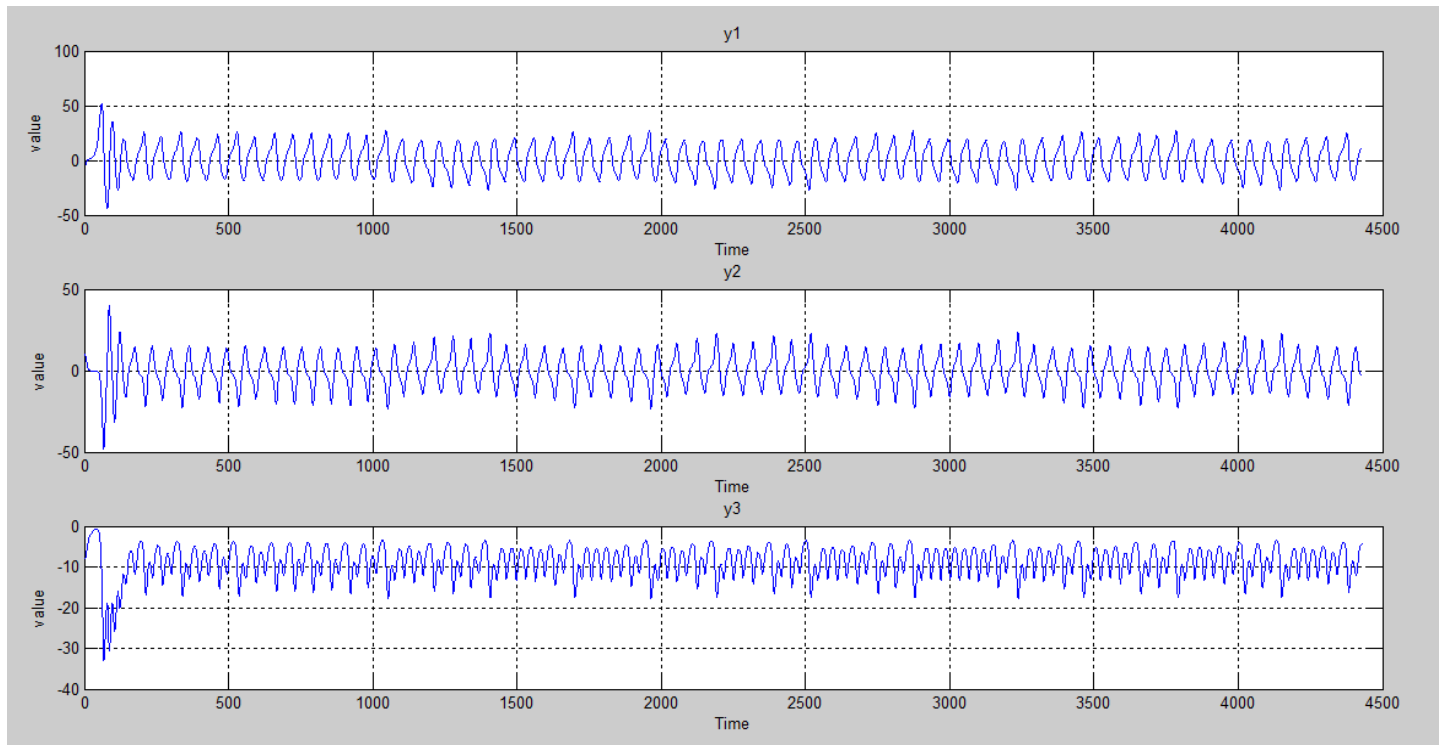

Figure 4: The Answer state chaotic attractor of the response system 


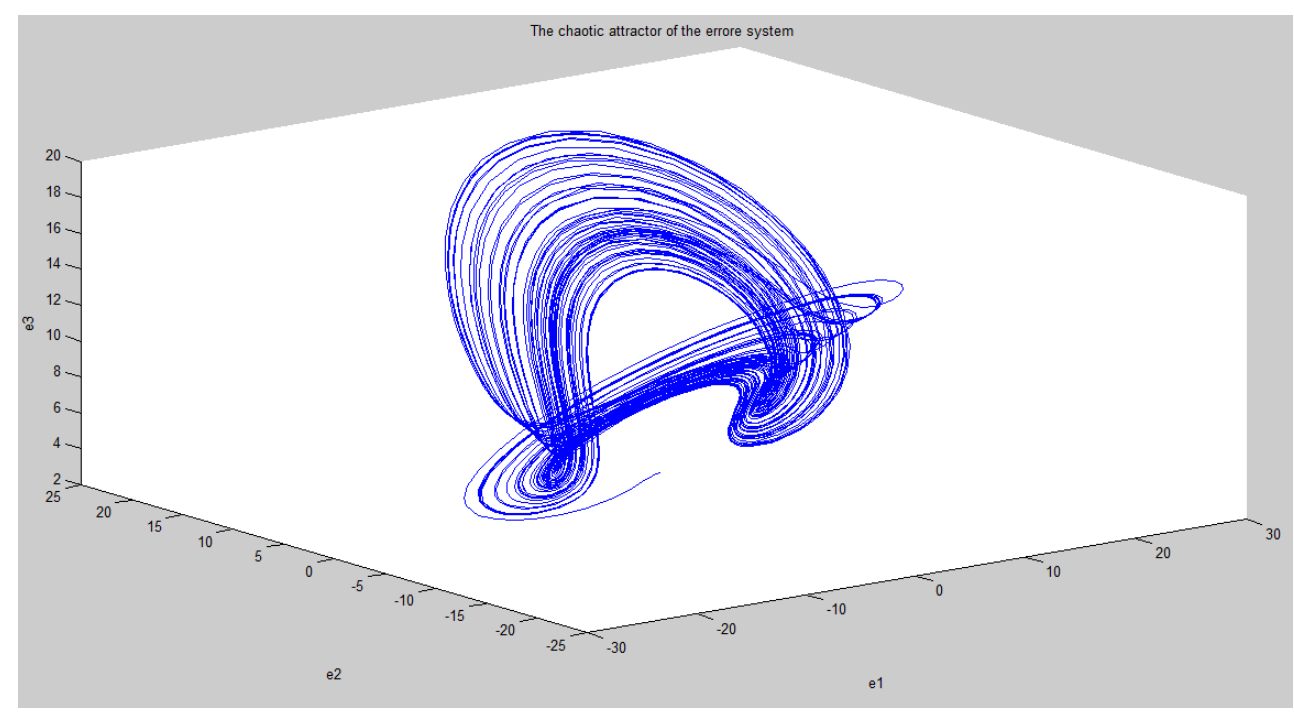

Figure 5: The chaotic attractor of the Fault chaotic system

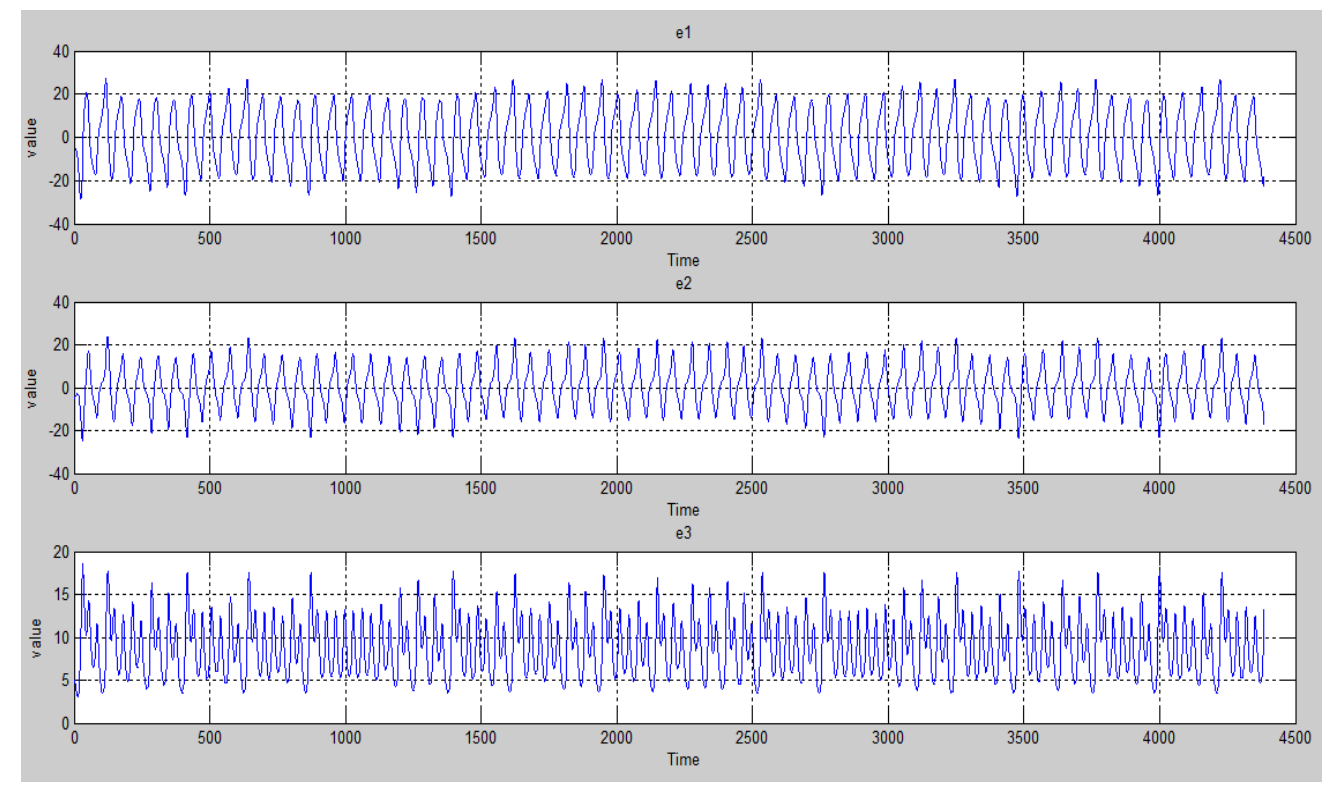

Figure 6: The Answer state chaotic attractor of the Fault chaotic system
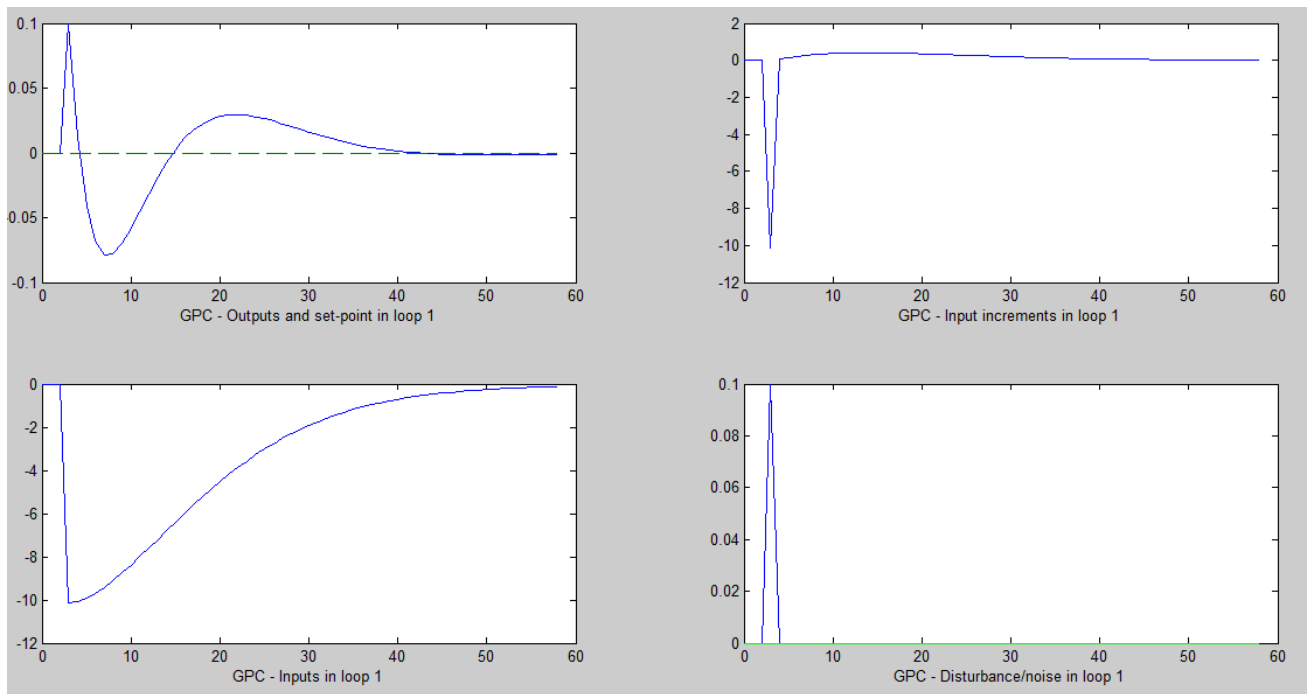

Figure 7: The output signal ' $y$ ', refrence signal' $r$ ' $, \Delta u, u$, noise , disturbance for The first controller 

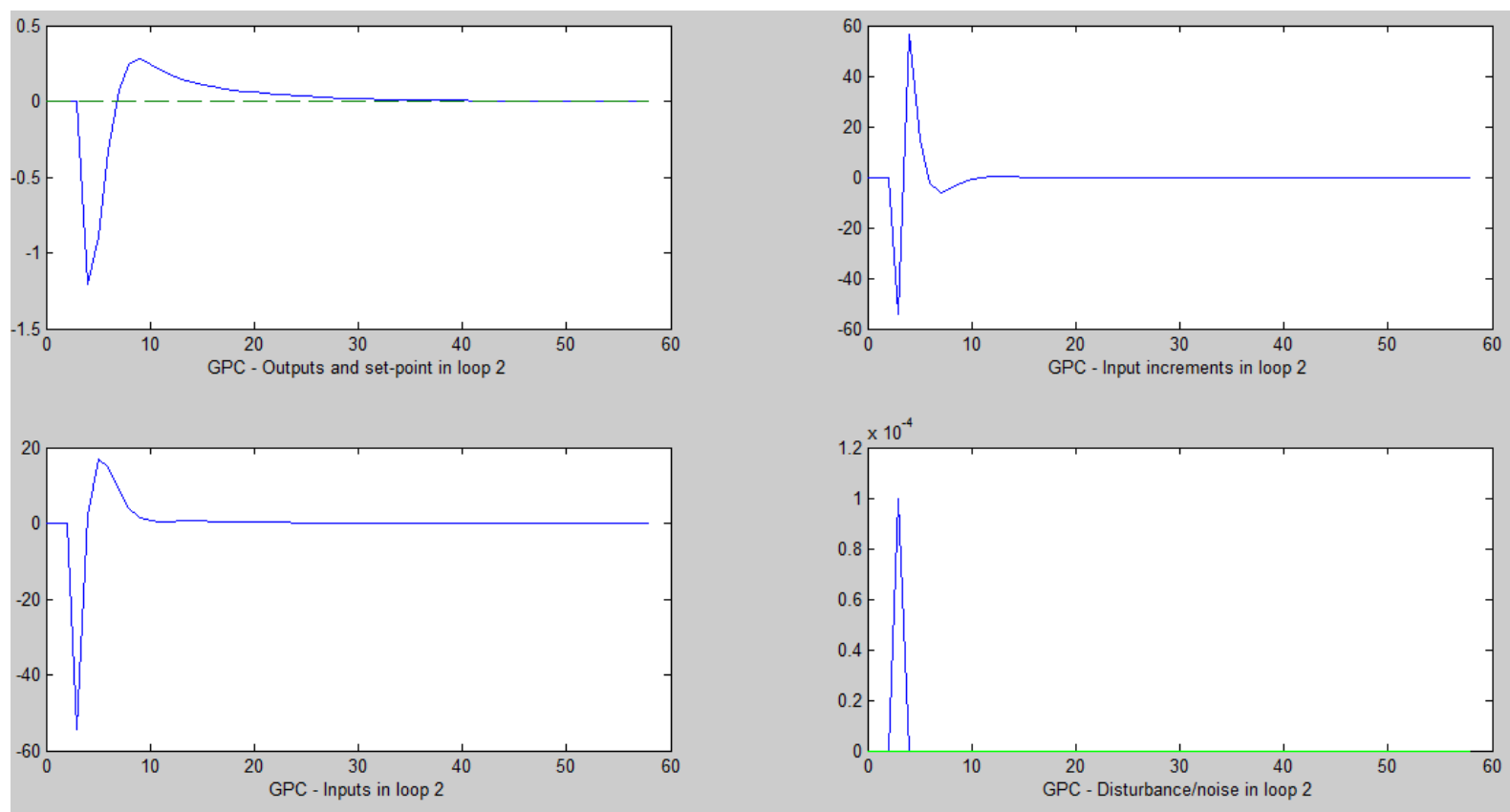

Figure 8: The output signal ' $y$ ', refrence signal' $r$ ' , $\Delta u, u$, noise , disturbance for second controller
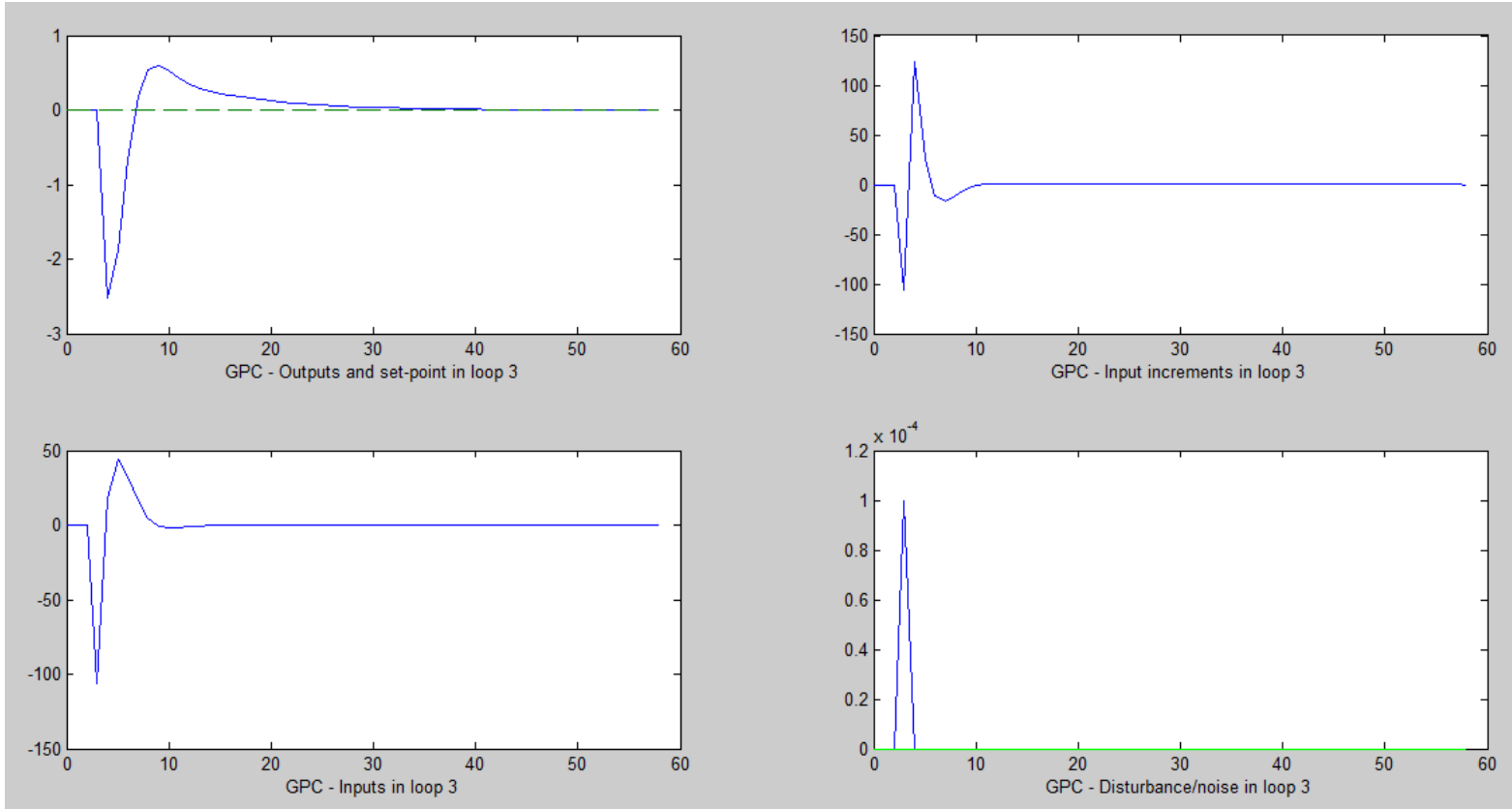

Figure 9: The output signal ' $y$ ', refrence signal' $r$ ' , $\Delta u, u$, noise , disturbance for third controller

\section{CONCLUSION}

Synchronization of chaos has been widely expanded as a major issue in the discussion of non-linear systems and is one of the most important subcategories of chaos control problem. Synchronization of today chaotic systems has lots of use in industry, laser industry, chemical reactors, secure telecommunications, cryptography and biology.

The aim of this study is synchronization of two Nadolschi chaotic systems. To do this the difference of two systems are obtained and called fault. If the fault of synchronization becomes zero it has been done correctly.

The controller that is used to stabilize the fault is generalized predictive controller. Generalized predictive controller has been designed from transfer function approach for modeling and based on linearization around the equilibrium. It has been presented that the controller can stabilize chaotic behavior and reduces it down to zero.

Considering that predictive controller has been used for synchronization and since predictive controller can see the future, therefore, due to the inherent characteristics, the controller can deal with system uncertainties.

\section{SUGGESTIONS FOR FUTURE WORK}

Synchronization of two Nadolschi super chaotic systems using generalized predictive control is among the suggestions that can be used in future $[6,7]$. 


\section{REFERENCES}

[1] L. M. Pecora and T. L. Carroll, Synchronization in chaotic systems, Phys. Rev. Lett., Vol.64, No.6, 821824, 1990.

[2] Y. X. Li, W. K. S. Tang, and G. R. Chen, Generating hyperchaos via state feedback control, Int J. of Bifur. \& Chaos, Vol.15, No.10, 3367-3375, 2005.

[3] A. M. Chen, J. N. Lu, J. H. L· u, and S. M. Yu, Generating hyperchaotic $\mathrm{L} \cdot \mathrm{u}$ attractor via state feedback control, Phys. A, Vol.364, No.1, 103-110, 2006.

[4] T. G. Gao, Z. Q. Chen, Z. Z. Yuan, and D. C. Yu, Adaptive synchronization of a new hyperchaotic system with uncertain parameters, Chaos, Solit. \& Fract., Vol.33, No.3, 922-928, 2007.

[5] J. C. Zhao and J. A. Lu, Using sampled-data feedback control and linear feedback synchronization in a new hyperchaotic system, Chaos, Solit. \& Fract., Vol.35, No.2, 376-382, 2008.
[6] Ruochen Zhang - and Yan Zhao - Hyperchaotic Attractors Generated from Nadolschi Chaotic System Springer ,Verlag Berlin Heidelberg - 2011 - 437- 444

[7] Yang, C.-D., Tao, C.-H., Wang, P - Comparison of feedback control methods for a hyper chaotic Lorenz system - Physics Letter A 374(5)-2010 - 729-732

[8] E.F.Camacho and C.Bordons "Model predictive control " Springer, Verlag London Limited, 2007.

[9] M. Pourmahmood, S. hanmohammadi, Gh. AlizadehSynchronization of two different uncertain chaotic systems with unknown parameters using a robust adaptive sliding mode controller-Elsevier - 2011

[10] L. Pecora, and T.Carroll, "Synchronization in chaotic systems", Code 6341, Naval Research Laboratory, Washington, D.C, 20375. USA, 1989.

[11] J.M.T Thompson, S.R Bishop, "Nonlinearity and Chaos in Engineering Dynamics", John Wiley \& Sons, $1995 .$. 